\title{
Organic light-emitting devices integrated with solar cells: High contrast and energy recycling
}

\author{
Chih-Jen Yang, Ting-Yi Cho, Chun-Liang Lin, and Chung-Chih $\mathrm{Wu}^{\mathrm{a})}$ \\ Department of Electrical Engineering, National Taiwan University, Taipei, Taiwan 10617, Republic of \\ China; Graduate Institute of Electro-Optical Engineering, National Taiwan University, Taipei, Taiwan 10617, \\ Republic of China and Graduate Institute of Electronics Engineering, National Taiwan University, \\ Taipei, Taiwan 10617, Republic of China
}

(Received 13 March 2007; accepted 28 March 2007; published online 25 April 2007)

\begin{abstract}
In this letter, the authors report that by integrating organic light-emitting devices (OLEDs) with solar cells, luminous ambient-light reflection as low as $1.4 \%$ (even superior to that achieved with polarizers) can be achieved without compromising the electroluminescence efficiency for high-contrast display applications. Furthermore, in such a configuration, the photon energies of the incident ambient light and the portion of OLED emission not getting outside of the device can be recycled into useful electrical power via the photovoltaic action, instead of being totally wasted as in other reported contrast-enhancement techniques. These features, the authors believe, shall make this technique attractive for high-contrast display applications and portable/mobile electronics that are highly power aware. (C) 2007 American Institute of Physics. [DOI: 10.1063/1.2732181]
\end{abstract}

Organic light-emitting devices (OLEDs), either conventional bottom emitting or top emitting, in general, are composed of a reflective back electrode, organic layers, and a (semi-)transparent exit electrode for light out coupling. ${ }^{1-3}$ With the reflective back electrode, OLEDs, in general, exhibit rather strong ambient-light reflection. Such reflection would seriously degrade the contrast of an OLED display under a strong lighting environment, since even at the off state the device will still exhibit some brightness due to ambient-light reflection. A few approaches have been used to reduce the ambient-light reflection and thus the contrast ratio of OLED displays, ${ }^{4-8}$ including (i) laminating polarizer or filter films on the surface of the display panel to reduce ambient-light reflection; (ii) inserting some layer(s) of optical purposes (absorption, interference, or both) into the devices to reduce ambient-light reflection. For all these contrast-enhancement approaches, the incident photon energies are simply absorbed and wasted. Moreover, in these contrast-enhancement structures, a significant portion of the internal OLED emission cannot come out and is also wasted.

Such wasted photon energies may be recycled by placing a solar cell in the back of an OLED. With such a structure, the incident ambient light, in principle, can be absorbed by the solar cell, thus reducing the reflection and giving higher contrast. Yet in contrast with previous contrast-enhancement approaches, the absorbed ambient light can now be converted to useful electrical power, instead of being totally wasted. The portion of internal OLED emission that cannot come out shall also be similarly absorbed by the solar cell and be converted to useful electrical power. Such a feature of energy recycling may be of significance and use for portable/ mobile electronics, since such devices typically are highly power aware. With such power recycling, the devices themselves would have the added self-powering function and the higher system-level power efficiency. Both could well contribute to longer battery lifetimes of portable/mobile electronics. In this letter, we report such integration of OLEDs and solar cells, which not only exhibits a contrast superior to

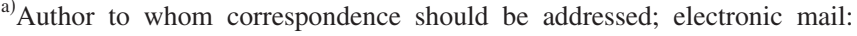
chungwu@cc.ee.ntu.edu.tw the conventional polarizer approach but also is capable of recycling both incident ambient illumination and internally generated OLED emission.

The device structure studied in this work is shown in Fig. 1, which consists of an inverted top-emitting OLED stacked back to back on top of an organic solar cell. A metal (e.g., Mo) in combination with indium tin oxide (ITO) $(40 \mathrm{~nm})$ was used as the bottom anode of the solar cell. Both ITO and Mo were deposited by magnetron sputtering. The organic solar cell had the conventional single donor-acceptor (D-A) heterostructure. ${ }^{9,10}$ Copper phthalocyanine $(30 \mathrm{~nm})$ was used as the donor and 3,4,9,10-perylenetetracarboxylic bis-benzimidazole $(50 \mathrm{~nm})$ was used as the acceptor. ${ }^{9,10} \mathrm{~A}$ thin layer $(18 \mathrm{~nm})$ of $\mathrm{Ag}$ was deposited on top of the D-A stack as the common semitransparent cathode for both the solar cell and the inverted OLED. This solar cell below the OLED plays dual roles, absorption and inducing destructive interference of the incident light, for largely reducing the ambient-light reflection.

The organic multilayers of the inverted top-emitting OLED in sequence consisted of tris-(8-hydroxyquinoline) aluminum $\left(\mathrm{Alq}_{3}\right)(10 \mathrm{~nm})$ doped with $20 \mathrm{wt} \%$ of $\mathrm{Cs}_{2} \mathrm{CO}_{3}$ as the electron injection layer, ${ }^{1,11,12}$ intrinsic $\mathrm{Alq}_{3}(50 \mathrm{~nm})$ as the electron-transport and emitting layer, $\alpha$-naphthyl phenylbiphenyl diamine ( $\alpha$-NPD) $(20 \mathrm{~nm})$ as the hole-transport

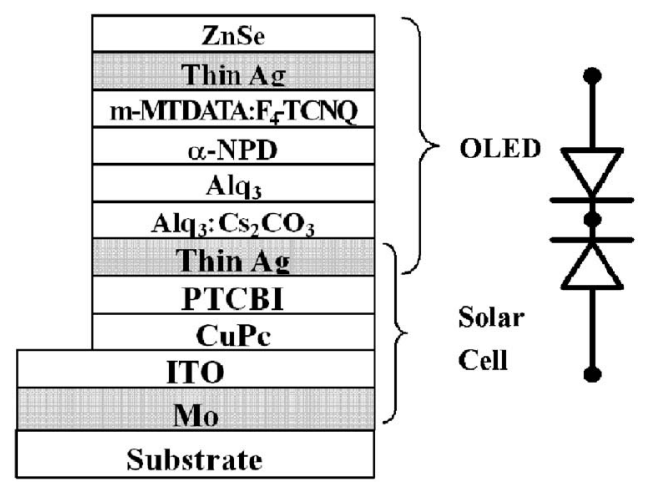

FIG. 1. Structure of the (OLED+solar cell) stack. 


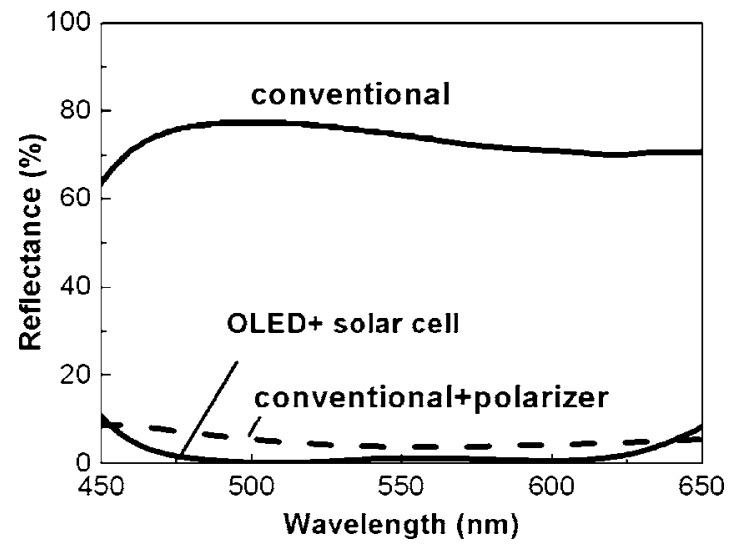

FIG. 2. Measured reflectance spectra of the (OLED+solar cell) stack, and the conventional bottom-emitting OLEDs with and without lamination with a circular polarizer film.

layer, ${ }^{4-8} 4,4^{\prime}, 4^{\prime \prime}$-tris(3-methylphenylphenylamino) triphenylamine ( $m$-MTDATA) $(30 \mathrm{~nm})$ doped with $2 \mathrm{wt} \%$ of tetrafluorotetracyano-quinodimethane as the hole-injection layer. ${ }^{13}$ Finally, a thin layer $(20 \mathrm{~nm})$ of $\mathrm{Ag}$ was deposited on top as the semitransparent anode of the inverted OLED. The thin layer of $\mathrm{Ag}$ with the thickness of $18-20 \mathrm{~nm}$ has relatively low optical absorption and high conductivity. The top $\mathrm{Ag}$ anode of OLED was further capped with a highindex dielectric layer of thermally evaporated $\mathrm{ZnSe}$ $(n \sim 2.4-2.5,30 \mathrm{~nm})$ as an index-matching layer to fine tune optical properties of the top electrode and the complete device stack. ${ }^{14,15}$ The thicknesses of the transparent ITO layer, organic layers, and the ZnSe layer had been carefully designed to optimize colors and efficiencies of the OLED and the reflection properties of the complete device stack. All the organic layers and the metal electrode layers were deposited in sequence by thermal evaporation without breaking the vacuum. After the device deposition, the devices were encapsulated with a cover glass under dry nitrogen atmosphere. The conventional bottom-emitting OLED with the structure of ITO anode $/ m$-MTDATA $(30 \mathrm{~nm}) / \alpha$-NPD $(20 \mathrm{~nm}) / \mathrm{Alq}_{3}$ $(60 \mathrm{~nm}) / \mathrm{LiF}(0.5 \mathrm{~nm}) / \mathrm{Al}$ cathode was also fabricated and tested in this study for comparison purposes.

Figure 2 shows the measured reflectance spectra of a conventional bottom-emitting device with or without lamination with a circular polarizer and the integrated (top-emitting OLED+solar cell) stack. The reflectance of the conventional OLED is high (up to $80 \%$ ) over the visible range, while that of the (OLED+solar cell) stack remains low over the whole visible range [even lower than that of a conventional (OLED+ polarizer) structure over most of the visible range]. The luminous reflectance $R_{L}$, the effective reflectance to human eyes under a light source with a spectral power distribution $S(\lambda)$, is defined as $R_{L}$ $=\int V(\lambda) S(\lambda) R(\lambda) d \lambda / \int V(\lambda) S(\lambda) d \lambda$, where $V(\lambda)$ is the standard photopic curve and $R(\lambda)$ is the reflectance spectrum. Here using $S(\lambda)$ of the standard illuminating source D65, one obtains a luminous reflectance of $\sim 1.4 \%$ for the (OLED + solar cell) stack, which is nearly two orders of magnitude lower than that $(70.6 \%)$ of the bottom-emitting device. Indeed such a luminous reflectance is even superior to that $(\sim 5 \%-6 \%)$ of the conventional (OLED+polarizer) structure. Photos in Fig. 3 show the appearance of a lowreflection (OLED+solar cell) stack (with one OLED on and Downloaded 18 Feb 2009 to 140.112 .113 .225 . Redistribution subje

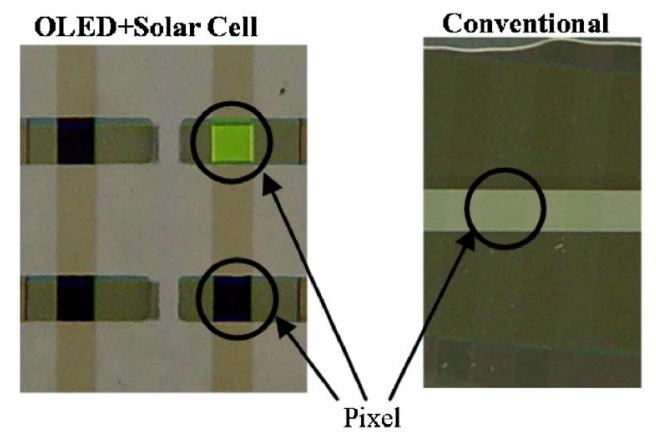

FIG. 3. (Color online) Photos of the (OLED+solar cell) stack and the conventional bottom-emitting OLED.

others off) and a highly reflective bottom-emitting OLED under strong ambient illumination. For the present (OLED + solar cell) stack, the high contrast between the off pixel (which is almost completely black) and the on pixel is clearly seen without using any further contrast-enhancement films.

Figure 4 shows the electroluminescence (EL) spectra measured at viewing angles of $0^{\circ}, 30^{\circ}$, and $60^{\circ}$ off the surface normal for the low-reflection energy-recycling topemitting OLED and that at $0^{\circ}$ for the conventional OLED. The emission spectra of the energy-recycling OLED are nearly the same as that of the conventional device and show hardly any shift with viewing angles. Furthermore, the measured emission pattern of the energy-recycling OLED is close to the ideal Lambertian, as shown in the inset of Fig. 4. Overall, through introducing the solar cell behind the OLED and through tuning the layer thicknesses in the structure, the present (OLED+solar cell) stack achieves the very broad low-reflection band and stable emission colors/brightness with viewing angles.

Figure 5 compares the brightness-current characteristics of the conventional bottom-emitting device and the lowreflection energy-recycling top-emitting device. The topemitting device exhibits an efficiency of $\sim 2.5 \mathrm{~cd} / \mathrm{A}$, which is about $50 \%$ of the cd/A efficiency of the conventional device ( $\sim 5 \mathrm{~cd} / \mathrm{A})$ and is slightly higher than that of the conventional OLED laminated with the circular polarizer $(\sim 2.1 \mathrm{~cd} / \mathrm{A})$.

Figure 6(a) shows the $I-V$ characteristics of the solar cell (beneath the OLED) both in the dark and under the AM1.5

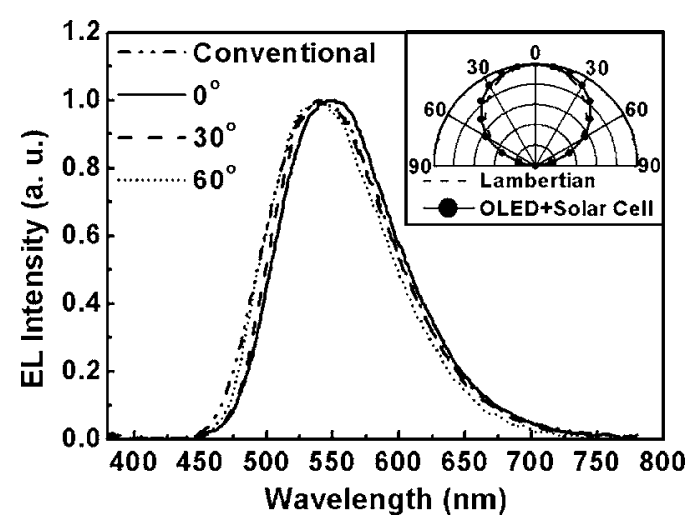

FIG. 4. EL spectra of the energy-recycling OLED at $0^{\circ}, 30^{\circ}$, and $60^{\circ}$ off the surface normal, along with the EL spectrum of the bottom-emitting OLED at $0^{\circ}$. Inset: the polar plot of the emission intensity of the energy-recycling OLED compared to the Lambertian pattern.

to AIP license or copyright; see http://apl.aip.org/apl/copyright.jsp 


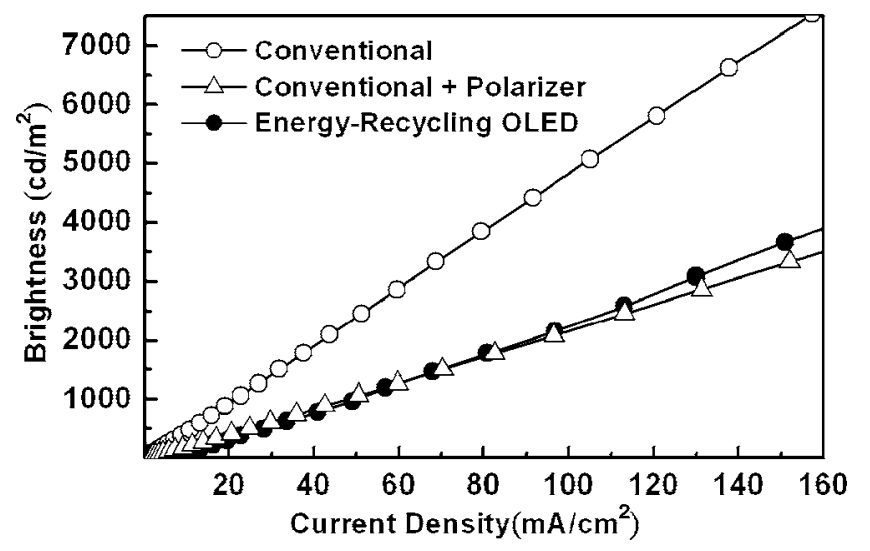

FIG. 5. Brightness-current characteristics of the energy-recycling OLED, and the conventional bottom-emitting OLED with or without lamination with a polarizer film.

illumination. In the inset of Fig. 6(a), the spectral response (external quantum efficiency as a function of the wavelength) of the device under the external illumination is shown. The solar cell shows a peak quantum efficiency of $\sim 6 \%$ around $600 \mathrm{~nm}$. The dark current (dashed line) of the solar cell follows well-behaved diode characteristics with a rectification ratio of $\sim 1 \times 10^{5}$ at $\pm 1 \mathrm{~V}$. As the device is illuminated at one sun with the AM1.5 solar simulator, the solar cell gives a short-circuit current $\left(I_{\mathrm{sc}}\right)$ of $1.35 \mathrm{~mA} / \mathrm{cm}^{2}$ and an open-circuit voltage $\left(V_{\mathrm{oc}}\right)$ of $0.4 \mathrm{~V}$. With the filling factor (FF) of $58 \%$, the maximum power conversion efficiency is $0.31 \%$. The power conversion efficiency achieved is about
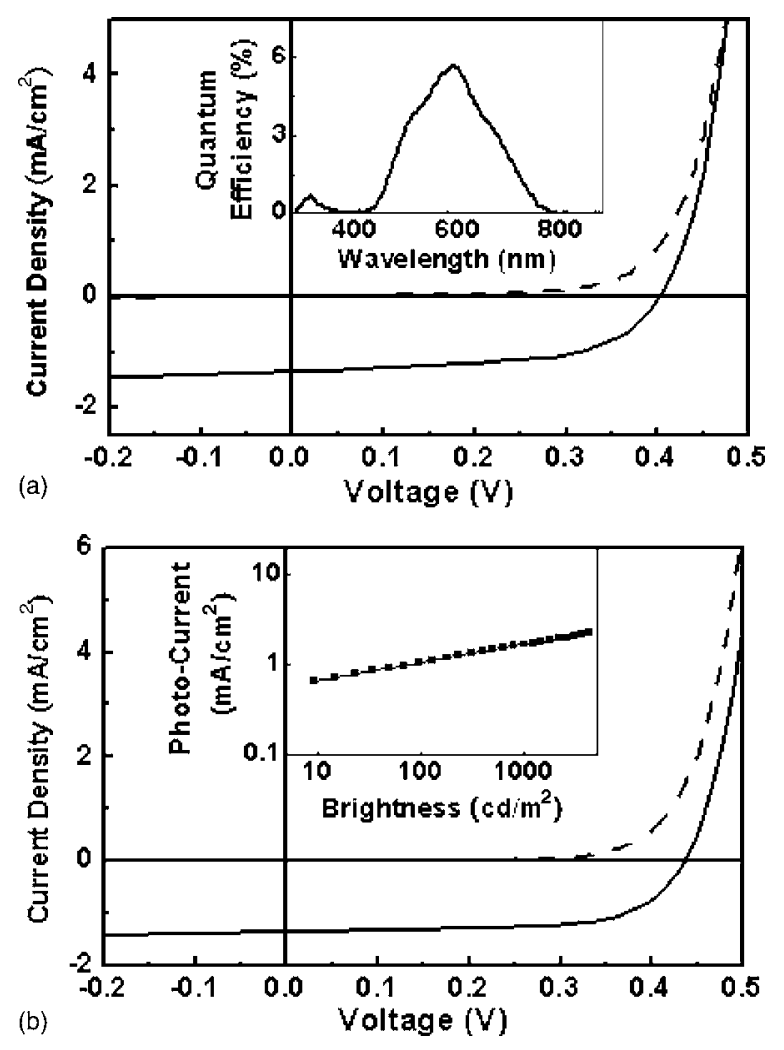

FIG. 6. (a) $I-V$ characteristics of the solar cell in dark and under the AM1.5 illumination. Inset of (a): Spectral response of the cell under external illumination. (b) $I-V$ characteristics of the solar cell in dark and under the illumination of the OLED above (driven at $20 \mathrm{~mA} / \mathrm{cm}^{2}, 315 \mathrm{~cd} / \mathrm{m}^{2}$ external brightness). Inset of (b): Photocurrent of the solar cell vs external brightness of the OLED. half of the efficiency achievable using the typical two-layer solar cell with the same organic materials. Yet, the device clearly shows the photovoltaic operation and the capability of recycling the incident ambient light.

Figure 6(b) shows the $I-V$ characteristics of the solar cell (beneath the OLED) in dark and under the illumination of the OLED above (driven at $20 \mathrm{~mA} / \mathrm{cm}^{2}$, corresponding to $315 \mathrm{~cd} / \mathrm{m}^{2}$ observed outside the device). The solar cell exhibits clear photovoltaic response when the OLED is turned on. The photocurrent of the solar cell shows a linear relationship with the external OLED brightness, as shown in the inset of Fig. 6(b). When driving the OLED at $20 \mathrm{~mA} / \mathrm{cm}^{2}$ $\left(315 \mathrm{~cd} / \mathrm{m}^{2}\right)$, the solar cell gives an $I_{\mathrm{sc}}$ of $1.36 \mathrm{~mA} / \mathrm{cm}^{2}$, a $V_{\mathrm{oc}}$ of $0.43 \mathrm{~V}$, and a FF of $65.5 \%$, corresponding to a maximum power of $0.38 \mathrm{~mW}$ recycled from the OLED operation and a power recycling efficiency (output power of solar cell/ electrical input power of OLED) of $0.26 \%$. This present recycling efficiency appears modest, yet one should notice that devices (both OLEDs and solar cells) used in this work are simply for the demonstration purpose and that there is plenty of room for further raising the recycling efficiency via using more efficient OLED and solar cell structures. Overall, results of Figs. 6(a) and 6(b) clearly show that the solar cell integrated with the OLED not only recycles the photon energy incident from the environment but also the photon energy generated internally from the OLED.

In summary, by integrating OLEDs with solar cells, luminous ambient-light reflection as low as $1.4 \%$ (even superior to that achieved with polarizers) can be achieved without compromising the EL efficiency for high-contrast display applications. Furthermore, in such a configuration, the photon energies of the incident ambient light and the portion of OLED emission not getting outside of the device can be recycled into useful electrical power via the photovoltaic action, instead of being totally wasted as in other reported contrast-enhancement techniques. These features shall make this present technique attractive for high-contrast display applications and portable/mobile electronics that are highly power aware.

${ }^{1}$ C. W. Tang and S. A. VanSlyke, Appl. Phys. Lett. 51, 913 (1987).

${ }^{2}$ M.-H. Lu, M. S. Weaver, T. X. Zhou, M. Rothman, R. C. Kwong, M. Hack, and J. J. Brown, Appl. Phys. Lett. 81, 3921 (2002).

${ }^{3}$ C.-C. Wu, P.-Y. Hsieh, C.-L. Lin, and H.-H. Chiang, Appl. Phys. Lett. 84, 3966 (2004).

${ }^{4}$ L. S. Hung and J. Madathil, Adv. Mater. (Weinheim, Ger.) 13, 1787 (2001).

${ }^{5}$ H. Aziz, Y.-F. Liew, H. M. Grandin, and Z. D. Popovic, Appl. Phys. Lett. 83, 186 (2003).

${ }^{6}$ S. H. Li, H. Liem, C. W. Chen, E. H. Wu, Z. Xu, and Y. Yang, Appl. Phys. Lett. 86, 143514 (2005).

${ }^{7}$ C.-J. Yang, C.-L. Lin, C.-C. Wu, Y.-H. Yeh, C.-C. Cheng, Y.-H. Kuo, and T.-H. Chen, Appl. Phys. Lett. 87, 143507 (2005).

${ }^{8}$ F. L. Wong, M. K. Fong, X. Jiang, C. S. Lee, and S. T. Lee, Thin Solid Films 446, 143 (2004).

${ }^{9}$ C. W. Tang, Appl. Phys. Lett. 48, 183 (1986).

${ }^{10}$ Larry D. Partain, Solar Cells and their Applications (Wiley, New York, 1995), p. 165-177. numbers

${ }^{11}$ C.-W. Chen, Y.-J. Lu, C.-C. Wu, E. H.-S. Wu, C.-W. Chu, and Y. Yang, Appl. Phys. Lett. 87, 241121 (2005).

${ }^{12}$ C.-I. Wu, C.-T. Lin, Y.-H. Chen, M.-H. Chen, Y.-J. Lu, and C.-C. Wu, Appl. Phys. Lett. 88, 152104 (2006).

${ }^{13}$ X. Zhou, M. Pfeiffer, J. Blochwitz, A. Werner, A. Nollau, T. Fritz, and K. Leo, Appl. Phys. Lett. 78, 410 (2001).

${ }^{14}$ H. Riel, S. Karg, T. Beierlein, B. Ruhstaller, and W. Rieß, Appl. Phys. Lett. 82, 466 (2003).

${ }^{15}$ C.-L. Lin, H.-W. Lin, and C.-C. Wu, Appl. Phys. Lett. 87, 021101 (2005). 